\title{
Il procedimento disciplinare notarile e la sua evoluzione storica
}

SOMMARIO: 1. Le origini antiche della figura del notaio. - 2. I tabelliones e

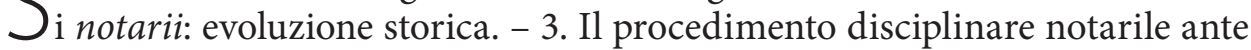
riforma. - 4. Il procedimento disciplinare notarile post riforma. -5 . Le ragioni della riforma del procedimento notarile. -6 . Il problema del contrasto della legge istitutiva della Co.Re.Di. con l'art. 102 Cost. - 7. L'organismo della Co.Re. Di.: la sua dislocazione territoriale. 8. La composizione della Co.Re.Di. - 9. La natura amministrativa del procedimento dinanzi alla Co.Re.Di.

1. Le origini antiche della figura del notaio. - La figura del notaio moderno ha origini storiche remote. Lo stesso lessico notarius, che, nei sistemi di notariato latino, designa ormai da secoli questa singolare categoria di pubblico ufficiale, di solito, si fa risalire al tempo della Repubblica romana antica (509-264 a.C.), nel cui ambito furono elaborati gli asserti embrionali dell'ordinamento del notariato ${ }^{1}$.

Nel diritto romano classico, per lo più, i notarii erano schiavi, pratici nell'uso delle notae tachigrafiche, sostanzialmente degli stenografi, a cui veniva affidata la scrittura di documenti con abbreviature, da parte di uomini politici, uomini d'affari, avvocati, scrittori e altri privati, che, anziché procedere alla stesura autografa delle minute, provvedevano alla composizione del testo scritto mediante il dictare ${ }^{2}$.

Soltanto nell'epoca successiva, durante il declino e la decadenza dell'Impero romano, nel periodo che va dal 180 d.C. al 1453, fino al 1590, secondo le linee tracciate nella famosa opera Storia del declino e della caduta dell'Impero romano

\footnotetext{
* Ferdinando Parente - Professore Ordinario di Diritto Civile, Diritto di Famiglia e Biodiritto Dipartimento Jonico in "Sistemi Giuridici ed Economici del Mediterraneo: società, ambiente, culture”, Università degli Studi di Bari “Aldo Moro”; ferdinando.parente@uniba.it

${ }^{1}$ Cfr. il sito Chi è il notaio, in www.consiglionotarilecosenza.it.

${ }^{2}$ G. Cencetti, Dal Tabellione Romano al Notaio Medievale, in scrineum.unipv.it.
} 
- pubblicata dallo storico inglese Edward Gibbon negli anni 1776 (vol. I), 1781 (vol. II-III) e 1788-1789 (vol. IV-V-VI) con il titolo originario The History of the Decline and Fall of the Roman Empire - il notarius assunse un ruolo vicino, ma non identico, a quello del notaio attuale, diventando, in un primo tempo, collaboratore dell'Imperatore e, successivamente, redattore di scritture su incarico e per conto delle parti ${ }^{3}$.

Nella diacronia della storia dell'umanità, figure assimilabili a quella del notarius sono rinvenibili sia nell'esperienza ebraica più risalente, in cui è testimoniata la presenza di scribi o segretari incaricati di ricevere contratti muniti di sigillo pubblico, sia nella vita sociale del popolo greco antico, che si avvaleva di scribi per ricevere e conservare contratti in funzione della prova della loro conclusione $e^{4}$.

2. I tabelliones $e$ i notarii: evoluzione storica. - In termini di connotazioni e funzioni, forse, le figure che più si avvicinano a quella del notaio moderno sono costituite dai tabelliones - così chiamati perché vergavano i loro atti su tavolette di cera -, i quali avevano il compito di redigere le scritture dei privati, curandone la forma legale: i loro atti, tuttavia, non godevano ancora di quella pubblica fede oggi attribuita agli atti pubblici redatti dai notai (art. 2699 c.c.) $)^{5}$.

Nel diritto romano, infatti, l'istrumento tabellionale era una mera scrittura, che godeva di una notevole considerazione, perché redatta da un esperto del diritto esercente l'attività sotto la vigilanza dello Stato, ma non aveva un valore assoluto. Soltanto i documenti emanati dalle magistrature e dagli uffici muniti del ius actorum conficiendorum godevano di piena fede $e^{6}$.

I tabelliones, già menzionati dal giurista Eneo Domizio Ulpiano (nato a Tiro il 170 circa e morto a Roma il 228), erano liberi professionisti, riuniti in collegi o scholae, tenuti ad osservare minute disposizioni redazionali e documentali, emanate soprattutto da Giustiniano negli anni 528, 536 e 538: Essi esercitavano l'attività in virtù di un'auctoritas, ossia di una concessione statale strettamente personale, di regola non delegabile, ed erano sottoposti alla sorveglianza della pubblica autorità?.

Solo in seguito, ai tempi di Carlo Magno, per effetto del Capitolare De scriviis et notariis dell'anno 805, si giunse alla fusione del tabellione e del notarius in un unico ufficio: gli atti del notaio acquistarono la stessa forza e gli stessi effetti delle sentenze passate in giudicato ${ }^{8}$.

${ }^{3}$ Cfr. la voce Declino e caduta dell'impero romano, in it.m.wikipedia.org.

${ }^{4}$ Cfr. il sito Chi è il notaio, in www.consiglionotarilecosenza.it.

${ }^{5}$ Cfr. il sito Chi è il notaio, in www.consiglionotarilecosenza.it.

${ }^{6}$ G. Cencetti, Dal Tabellione Romano al Notaio Medievale, in scrineum.unipv.it.

${ }^{7}$ G. Cencetti, Dal Tabellione Romano al Notaio Medievale, in scrineum.unipv.it.

${ }^{8}$ Cfr. il sito Chi è il notaio, in www.consiglionotarilecosenza.it. 
Nel Medioevo, tra i secoli XI e XII, per effetto della piena attribuzione agli atti notarili della publica fides, la professione del notaio iniziò ad essere reputata prestigiosa e autorevole. Tuttavia, solo nel periodo della Rivoluzione francese (1789-1799) si delinearono gli assetti essenziali della figura del notaio moderno, sottoposta ad una rigorosa vigilanza pubblica e ad un peculiare procedimento disciplinare ${ }^{9}$.

In Italia, la figura del notaio e il procedimento notarile furono disciplinati, per la prima volta, dalla 1.25 luglio 1875, n. 2786, poi, dal Regio decreto 25 maggio 1879, n. 4900, e, infine, dalla l. 16 febbraio 1913, n. 89, tuttora in vigore, sia pure con rilevanti modifiche ed integrazioni ${ }^{10}$.

3. Il procedimento disciplinare notarile ante riforma. - Nel testo originario della legge n. 89 del 1913, il procedimento disciplinare notarile era modulato sulla diversificazione delle competenze, secondo la gravità delle sanzioni ${ }^{11}$ : per le sanzioni disciplinari più gravi, quali l'ammenda, la sospensione e la destituzione, la competenza per i tre gradi di giudizio era affidata al giudice ordinario: Tribunale, Corte d'Appello e Corte di Cassazione (artt. 151-156, 1. 16 febbraio 1913, n. 89); per le sanzioni meno gravi, ossia l'avvertimento e la censura, la competenza, soltanto nel primo grado, era conferita al Consiglio notarile distrettuale, che decideva con provvedimento impugnabile dinanzi al giudice ordinario ${ }^{12}$.

La sentenza del giudice di secondo grado, a sua volta, non era appellabile nel merito (artt. 148-150, 1. 16 febbraio 1913, n. 89), ma poteva essere impugnata per motivi di legittimità davanti alla Corte di Cassazione, ex art. 111 Cost. ${ }^{13}$.

Il d.lgs. 1 agosto 2006, n. 249 ha modificato radicalmente il regime del procedimento disciplinare notarile $\mathrm{e}^{14} \mathrm{e}$ ha istituito la Commissione amministrativa regionale di disciplina (c.d. Co.Re.Di.), per l'applicazione delle sanzioni

${ }^{9}$ Cfr. il sito Chi è il notaio, in www.consiglionotarilecosenza.it.

${ }^{10}$ Cfr. il sito Chi è il notaio, in www.consiglionotarilecosenza.it.

${ }^{11}$ F. Parente, Il procedimento disciplinare notarile, in P. Boero e M. Ieva (a cura di), La legge notarile, Milano, 2014, p. 823; S. Caporusso, Il nuovo procedimento disciplinare notarile. Lineamenti, in S. Pagliantini, (a cura di), Il diritto vivente nell'età dell'incertezza. Saggi sull'art. 28 ed il procedimento disciplinare riformato, $2^{\mathrm{a}}$ ed., Torino, 2012, 239. Sull'attualità della gerarchia delle sanzioni, in funzione della graduazione della gravità, v. Corte Cass., 23 marzo 2012, n. 4720, in $h t t p: / / w w w$. dejure.giuffre.it.

12 F. Parente, Il procedimento disciplinare notarile, cit., pp. 823-824; M. Di Fabio, Manuale di notariato, $2^{\text {a }}$ ed., Milano, 2007, p. 392; A. Ciatti, Il procedimento disciplinare a carico dei notai: la prescrizione, la decadenza e il diritto transitorio, in Rass. dir. civ., 2011, 256 ss.; S. Caporusso, Il nuovo procedimento disciplinare notarile. Lineamenti, cit., p. 241.

${ }^{13}$ F. Parente, Il procedimento disciplinare notarile, cit., p. 824; P. Boero, La legge notarile commentata con la dottrina e la giurisprudenza, II, Torino, 1993, p. 640; E. Protettì e C. Di Zenzo, La legge notarile, $5^{\text {a }}$ ed., Milano, 2009, p. 474 ss.

${ }^{14}$ F. Parente, Il procedimento disciplinare notarile, cit., p. 823; R. Danovi, Il procedimento disciplinare dei notai avanti la CO.RE.DI. tra norme di legge e principi di diritto, in N, 2013, 338; 
disciplinari e delle misure cautelari nei confronti dei notai e per la valutazione dei presupposti di cessazione temporanea o definitiva dalle funzioni notarili, nei casi previsti dall'art. 34, comma 2, 1. n. 89 del $1913^{15}$.

Il d.P.R. 7 agosto 2012, n. 137, sul «procedimento disciplinare delle professioni regolamentate», ha ribadito che, malgrado la novazione normativa, «restano ferme le disposizioni vigenti in materia disciplinare concernenti la professione di notaio (art. 8, comma 4) ${ }^{16}$.

La sanzione disciplinare dell'avvertimento, dunque, continua a trovare applicazione per tutte le contravvenzioni prive di specifica sanzione, come si desume dal parere del Consiglio di Stato in data 16 marzo 2006 e dalla circolare del Ministero della Giustizia in data 23 agosto 2006, n. 10/2006. In altri termini, il legislatore delegato ha confermato il carattere residuale della sanzione dell'avvertimento pure nel nuovo regime delle fonti ${ }^{17}$.

4. Il procedimento disciplinare notarile post riforma. - A seguito della riforma del 2006, che ha modificato gli artt. 148 ss. della legge notarile, il Consiglio notarile distrettuale ha conservato il potere di gestire la sola fase d'indagine, finalizzata all'eventuale promozione dell'azione disciplinare dinanzi alla Co.Re.Di., che è l'organo legittimato allo svolgimento del giudizio di primo grado, per tutte le sanzioni disciplinari ${ }^{18}$, e che decide con provvedimento impugnabile innanzi alla Corte d'Appello ${ }^{19}$, che, a sua volta, emette una sentenza oppugnabile dinanzi alla Corte di Cassazione ${ }^{20}$.

Al Consiglio notarile distrettuale, dunque, il legislatore ha assegnato sia il ruolo d'indagine e di controllo sul regolare svolgimento dell'attività notarile,

S. Caporusso, Il nuovo procedimento disciplinare notarile. Lineamenti, cit., p. 240; G. Santarcangelo, Il procedimento disciplinare a carico dei notai. Decreto legislativo 1 agosto 2006, Milano, 2007, passim.

${ }^{15}$ F. Parente, Il procedimento disciplinare notarile, cit., p. 823; S. Caporusso, Il nuovo procedimento disciplinare notarile. Lineamenti, cit., p. 239 ss.

${ }^{16}$ R. Danovi, Il procedimento disciplinare dei notai avanti la CO.RE.DI. tra norme di legge e principi di diritto, cit., p. 338.

${ }_{17}$ Cfr. Co.Re.Di. Lombardia, 7 febbraio 2013, in Notariato, 2013, p. 348; contra, Corte Cass., Sez. VI, 24 luglio 2012, n. 12995, in CED Cassazione.

${ }_{18}$ M. Di Fabio, Manuale di notariato, cit., pp. 392-393; R. Danovi, Il procedimento disciplinare dei notai avanti la CO.RE.DI. tra norme di legge e principi di diritto, in Notariato, 2013, 338; S. Caporusso, Il nuovo procedimento disciplinare notarile. Lineamenti, cit., p. 240.

19 R. Danovi, Il procedimento disciplinare dei notai avanti la CO.RE.DI. tra norme di legge e principi di diritto, in Notariato, 2013, p. 338; S. Caporusso, Il nuovo procedimento disciplinare notarile. Lineamenti, cit., pp. 241-242.

${ }^{20}$ F. Parente, Il procedimento disciplinare notarile, cit., p. 824; S. Caporusso, Il nuovo procedimento disciplinare notarile. Lineamenti, cit., p. 242. 
sia il potere di attivare il procedimento disciplinare, tramite il diritto di azione accreditato al suo presidente ${ }^{21}$.

5. Le ragioni della riforma del procedimento notarile. - Nell'àmbito della categoria dei notai, il proposito di riformulare il regime del procedimento disciplinare è giunto a maturazione sulla base di un duplice ordine di ragioni: a) per accreditare l'irrogazione delle sanzioni disciplinari, comprese quelle di maggiore gravità, ad un organismo interno alla categoria dei notai, in analogia alle opzioni già in vigore per altre categorie professionali; $b$ ) per adeguare la misura dell'ammenda all'attualità dei valori monetari, onde rendere congrua la sanzione pecuniaria in sede di procedimento disciplinare 22 .

La riforma del procedimento disciplinare ha comportato un delicato lavoro di bilanciamento tra le esigenze di organizzazione della professione notarile e la funzione pubblica esercitata dai notai ${ }^{23}$.

Difatti, se, da un lato, il legislatore ha attribuito ad un organismo interinale alla categoria il controllo sull'attività notarile ${ }^{24}$, dall'altro, ha riservato un ruolo non marginale alla magistratura ordinaria, in conformità con le connotazioni d'interesse generale della funzione notarile $e^{25}$.

Per di più, in una prospettiva sistematica, la nuova articolazione del regime del procedimento disciplinare ha posto all'interprete l'onere di valutare se l'istituzione di un organismo di controllo di categoria non sia in contrasto con i canoni assiologici sottesi all'art. 102, comma 2, Cost., che vieta la costituzione di organismi speciali di giurisdizione ${ }^{26}$.

${ }^{21}$ F. Parente, Il procedimento disciplinare notarile, cit., p. 824; G. Casu, Sull'intervento del Consiglio notarile nei procedimenti disciplinari a carico di notai, in Riv. not., 2007, II, 1197 ss.; R. Danovi, Il procedimento disciplinare dei notai avanti la CO.RE.DI. tra norme di legge e principi di diritto, in Notariato, 2013, p. 338; S. Caporusso, Il nuovo procedimento disciplinare notarile. Lineamenti, cit., pp. 241-242.

${ }^{22}$ F. Parente, L'istituzione dell'organo del giudizio disciplinare (Co.Re.Di.), in P. Boero e M. Ieva (a cura di), La legge notarile, cit., p. 824; R. Danovi, Il procedimento disciplinare dei notai avanti la CO.RE.DI. tra norme di legge e principi di diritto, in Notariato, 2013, pp. 338-339; S. Caporusso, Il nuovo procedimento disciplinare notarile. Lineamenti, cit., p. 239 ss.

${ }^{23}$ F. Parente, L'istituzione dell'organo del giudizio disciplinare (Co.Re.Di.), cit., p. 824; M. Di Fabio, Manuale di notariato, cit., p. 392 ss.

${ }^{24}$ M. Bove, Considerazioni in merito al procedimento disciplinare notarile, in Vita not., 2014, p. 418; S. Caporusso, Il nuovo procedimento disciplinare notarile. Lineamenti, cit., p. 240.

${ }^{25}$ F. Parente, L'istituzione dell'organo del giudizio disciplinare (Co.Re.Di.), cit., p. 824; G. Casu e G. Sicchiero, La legge notarile commentata, Torino, 2010, p. 556.

${ }^{26}$ F. Parente, L'istituzione dell'organo del giudizio disciplinare (Co.Re.Di.), cit., pp. 824-825; E. Fabiani, Il nuovo procedimento disciplinare notarile, in GPC, 2009, p. 87 ss. 
6. Il problema del contrasto della legge istitutiva della Co.Re.Di. con l'art. 102 Cost. - La dottrina ha prospettato il plausibile contrasto tra l'art. 102 Cost. e la legge istitutiva della Co.Re.Di.

Per confutare il contrasto è stato rilevato che l'attività di cognizione e d'irrogazione delle sanzioni disciplinari, da parte dell'organismo notarile, non è un'operazione giurisdizionale, ma una funzione assimilabile all'azione amministrativa ${ }^{27}$.

In realtà, l'incertezza sulla natura delle funzioni che la giurisdizione domestica è chiamata a svolgere ha portato a preservare un certo potere di controllo e di decisione in capo all'autorità giurisdizionale, a maggiore tutela dell'attività notarile e della sua funzione pubblica ${ }^{28}$.

L'esigenza di contemperare gli interessi professionali della categoria dei notai con il rispetto della funzione solenne dell'attività notarile, nell'architettura complessiva del procedimento disciplinare e nell'articolazione delle competenze, ha spinto il legislatore a prevedere soltanto un primo grado di giudizio dinanzi alla Co.Re.Di. ${ }^{29}$, mentre lo ha indotto a riservare agli organismi della giurisdizione ordinaria il compito d'intervenire nei gradi successivi, in caso di impugnazione del provvedimento emesso dall'organo di giudizio interinale, per valutare giudizialmente la congruità della sanzione in concreto erogata nel primo grado ${ }^{30}$.

Tuttavia, la competenza sui vizi della fase istruttoria, che precede l'avvio del procedimento, non rientra nella giurisdizione del giudice amministrativo, ma spetta al giudice ordinario, a cui è riservato il sindacato sulle decisioni in materia di infrazioni disciplinari notarili.

Difatti, ai fini della giurisdizione, non sussiste una ragione plausibile per separare l'iniziativa disciplinare dal procedimento, posto che l'eventuale illegittimità dell'avvio della procedura non ha natura diversa da quella concernente gli atti successivi.

In realtà, pur trattandosi di atti di un procedimento amministrativo, in entrambi i casi siamo in presenza di diritti soggettivi, la cui tutela, come sempre accade nel campo disciplinare, è devoluta al giudice ordinario ${ }^{31}$.

${ }_{27}$ F. Parente, L'istituzione dell'organo del giudizio disciplinare (Co.Re.Di.), cit., p. 825; S. Caporusso, Il nuovo procedimento disciplinare notarile. Lineamenti, cit., p. 240; G. Casu e G. Sicchiero, La legge notarile commentata, cit., p. 556.

${ }^{28}$ F. Parente, L'istituzione dell'organo del giudizio disciplinare (Co.Re.Di.), cit., p. 825; E. Fabiani, Il nuovo procedimento disciplinare notarile, in GPC, 2009, p. 87 ss.; M. Molinari, A due anni dalla riforma del disciplinare notarile, Notariato, 2009, p. 467 ss.

${ }^{29}$ G. Casu, in G. Mariconda, G. Casu e V. Tagliaferri, Codice della legge notarile, Torino, 2013, p. 439.

${ }^{30}$ F. Parente, L'istituzione dell'organo del giudizio disciplinare (Co.Re.Di.), cit., p. 825; S. Caporusso, Il nuovo procedimento disciplinare notarile. Lineamenti, cit., p. 240 ss.

${ }^{31}$ F. Parente, L'istituzione dell'organo del giudizio disciplinare (Co.Re.Di.), cit., p. 825; M. Miccoli, Due nodi da sciogliere: prescrizione del procedimento disciplinare e illeciti disciplinari non codificati, 
7. L'organismo della Co.Re.Di.: la sua dislocazione territoriale. - Dal punto di vista della dislocazione sul territorio nazionale, la Co.Re.Di. è istituita in ogni circoscrizione territoriale e ha sede presso il consiglio notarile distrettuale del capoluogo della regione ${ }^{32}$.

La Valle d'Aosta ed il Piemonte, le Marche e l'Umbria, l'Abruzzo ed il Molise, la Campania e la Basilicata, il Trentino-Alto Adige, il Friuli-Venezia Giulia ed il Veneto formano uniche circoscrizioni territoriali con sede, rispettivamente, presso il Consiglio notarile distrettuale del capoluogo delle regioni Piemonte, Marche, Abruzzo, Campania e Veneto ${ }^{33}$.

Qualora il territorio di un distretto ricada in due diverse Regioni, l'intero territorio si considera compreso nella circoscrizione in cui è ubicato il maggior numero di sedi dello stesso distretto. Infine, i distretti riuniti di La Spezia e Massa sono compresi nella circoscrizione della Liguria ${ }^{34}$.

8. La composizione della Co.Re.Di. - La Co.Re.Di. è composta da un magistrato, che la presiede, e da sei, otto o dodici notai, a seconda che il numero dei notai assegnati a ciascuna circoscrizione non sia superiore a duecentocinquanta o risulti superiore a tale numero, ma inferiore a quattrocento, ovvero sia pari o superiore a quattrocento ${ }^{35}$.

Ai sensi degli artt. 150 e 151-bis della legge notarile, di regola, possono essere eletti componenti della commissione tutti i notai iscritti ai collegi dei distretti di ciascuna circoscrizione territoriale.

I membri della commissione, compresi il segretario e il tesoriere, durano in carica tre anni ${ }^{36}$ e, per prorogatio legis, svolgono le loro funzioni fino all'insediamento dei nuovi componenti ${ }^{37}$.

Infine, le spese per l'elezione della componente notarile della commissione, i costi per il funzionamento, per i locali della sede, per il personale, per le attrezzature, per i rimborsi e i gettoni di presenza e tutte le altre spese necessarie

Notariato, 2013, p. 228. In giurisprudenza, cfr. Corte Cass., Sez. un, 31 luglio 2012, n. 13617, in CED Cassazione.

32 F. Parente, L'istituzione dell'organo del giudizio disciplinare (Co.Re.Di.), cit., p. 825; S. Caporusso, Il nuovo procedimento disciplinare notarile. Lineamenti, cit., p. 240; M. Di Fabio, Manuale di notariato, cit., p. 393.

${ }^{33}$ F. Parente, L'istituzione dell'organo del giudizio disciplinare (Co.Re.Di.), cit., p. 825; M. Di Fabio, Manuale di notariato, cit., p. 393.

${ }^{34}$ F. Parente, L'istituzione dell'organo del giudizio disciplinare (Co.Re.Di.), cit., p. 825; M. Di Fabio, Manuale di notariato, cit., p. 394.

${ }_{35}$ F. Parente, L'istituzione dell'organo del giudizio disciplinare (Co.Re.Di.), cit., p. 825; M. Di Fabio, Manuale di notariato, cit., p. 394.

${ }^{36}$ S. Caporusso, Il nuovo procedimento disciplinare notarile. Lineamenti, cit., p. 240; M. Di Fabio, Manuale di notariato, cit., p. 394.

${ }^{37}$ F. Parente, L'istituzione dell'organo del giudizio disciplinare (Co.Re.Di.), cit., pp. 825-826. 
all'attività della commissione sono sostenute dai consigli notarili distrettuali di ciascuna circoscrizione territoriale ${ }^{38}$, in proporzione agli onorari repertoriali percepiti dai notai aventi sede nella stessa circoscrizione nell'anno precedente, e imputate annualmente ai singoli notai dei distretti nel computo dell'importo complessivo della tassa collegiale ${ }^{39}$.

9. La natura amministrativa del procedimento dinanzi alla Co.Re.Di. - La definizione della natura amministrativa o giurisdizionale del procedimento dinanzi alla Co.Re.Di., modulato sul principio accusatorio ${ }^{40}$, ha rilevanza per l'inquadramento del procedimento disciplinare notarile nell'alveo delle procedure amministrative o nel sistema dei procedimenti giurisdizionali ${ }^{41}$.

La collocazione del procedimento notarile nell'uno o nell'altro àmbito, infatti, implica l'applicazione o la disapplicazione del regime della 1.7 agosto 1990, n. 241 e sue modifiche, in materia di procedimento amministrativo, ovvero delle norme del codice di procedura civile o penale, e condiziona la proposizione di eccezioni di illegittimità costituzionale ${ }^{42}$.

In realtà, la natura amministrativa dell'organismo della Co.Re.Di., conseguente al divieto di istituzione di giudici speciali (art. 102, comma 2, Cost.) ${ }^{43}$, depone per la qualificazione amministrativa del procedimento disciplinare notarile di primo grado ${ }^{44}$, con tutte le induzioni e preclusioni normative ${ }^{45}$.

La qualificazione comporta la nomina del responsabile del procedimento, che, per esigenze di celerità nell'adozione della decisione, dovrebbe competere al presidente, anziché all'intero collegio ${ }^{46}$, e la configurazione della decisione

38 M. Di Fabio, Manuale di notariato, cit., p. 393.

39 F. Parente, L'istituzione dell'organo del giudizio disciplinare (Co.Re.Di.), cit., p. 826.

40 Sul punto, cfr. Corte Cass., 27 maggio 2011, n. 11790, in Notariato, 2012, pp. 223-224.

${ }^{41}$ F. Parente, La natura del procedimento dinanzi alla Co.Re.Di., in P. Boero e M. Ieva (a cura di), La legge notarile, cit., p. 826; M. Bove, Considerazioni in merito al procedimento disciplinare notarile, cit., p. 419; S. Caporusso, Il nuovo procedimento disciplinare notarile. Lineamenti, cit., p. 239 ss.; V. Tenore e G. Celeste, La responsabilità disciplinare del notaio ed il relativo procedimento, Milano, 2008, p. 10 ss. e p. 155 ss.; A. Brienza, Il nuovo procedimento disciplinare, in Federnotizie, 16/2007, p. 54.

${ }^{42}$ F. Parente, La natura del procedimento dinanzi alla Co.Re.Di., cit., p. 826.

${ }_{43}$ M. Bove, Considerazioni in merito al procedimento disciplinare notarile, cit., p. 419; G. Casu e G. Sicchiero, La legge notarile commentata, cit., p. 541 ss.

44 R. Danovi, Il procedimento disciplinare dei notai avanti la CO.RE.DI. tra norme di legge e principi di diritto, cit., p. 339; S. Caporusso, Il nuovo procedimento disciplinare notarile. Lineamenti, cit., p. 240; G. Casu e G. Sicchiero, La legge notarile commentata, cit., p. 541 ss. In senso contrario, cfr. A. Brienza, Il nuovo procedimento disciplinare, cit., p. 54.

45 F. Parente, La natura del procedimento dinanzi alla Co.Re.Di., cit., p. 826.

${ }^{46}$ M. Bove, Considerazioni in merito al procedimento disciplinare notarile, cit., p. 420; F.C. Rampulla, I principi generali della legge n. 241/1990 ed il responsabile del procedimento, in FA, $2008,641$. 
come provvedimento amministrativo, suscettibile di revoca (art. 21-quinquies, 1. n. 241/1990) e di annullamento in autotutela (art. 21-nonies, 1. n. 241/1990) ${ }^{47}$.

\section{Bibliografia:}

Boero P., La legge notarile commentata con la dottrina e la giurisprudenza, II, Torino, 1993, p. 640; Bove M., Considerazioni in merito al procedimento disciplinare notarile, in Vita not., 2014, p. 418; Brienza A., Il nuovo procedimento disciplinare, in Federnotizie, 16/2007, p. 54.

Caporusso S., II nuovo procedimento disciplinare notarile. Lineamenti, in S. PAGLIANTINI, (edited by), Il diritto vivente nell'età dell'incertezza. Saggi sull'art. 28 ed il procedimento disciplinare riformato, $2^{\text {a }}$ ed., Torino, 2012, 239.

Cass., 23 marzo 2012, n. 4720, in http://www.dejure.giuffre.it.

Cass., Sez. VI, 24 luglio 2012, n. 12995, in CED Cassazione.

Cass., Sez. un, 31 luglio 2012, n. 13617, in CED Cassazione.

Casu G. e Sicchiero G., La legge notarile commentata, Torino, 2010, p. 556.

Casu G., in G. Mariconda, G. Casu e V. Tagliaferri, Codice della legge notarile, Torino, 2013, p. 439.

Casu G., Sull'intervento del Consiglio notarile nei procedimenti disciplinari a carico di notai, in Riv. not., 2007, II, 1197 ss.

Cencetti G., Dal Tabellione Romano al Notaio Medievale, in scrineum.unipv.it.

Chi è il notaio, in www.consiglionotarilecosenza.it.

Ciatti A., Il procedimento disciplinare a carico dei notai: la prescrizione, la decadenza e il diritto transitorio, in Rass. dir. civ., 2011, 256 ss.

Co.Re.Di. Lombardia, 7 febbraio 2013, in Notariato, 2013, p. 348.

Danovi R., Il procedimento disciplinare dei notai avanti la CO.RE.DI. tra norme di legge e principi di diritto, in N, 2013, 338.

Declino e caduta dell'impero romano, in it.m.wikipedia.org.

Di Fabio M., Manuale di notariato, 2a ed., Milano, 2007, p. 392.

Fabiani E., II nuovo procedimento disciplinare notarile, in GPC, 2009, p. 87 ss.

Miccoli M., Due nodi da sciogliere: prescrizione del procedimento disciplinare e illeciti disciplinari non codificati, Notariato, 2013, p. 228.

Molinari M., A due anni dalla riforma del disciplinare notarile, Notariato, 2009, p. 467 ss.

PARENTE F., II procedimento disciplinare notarile, in P. BOERO e M. IEVA (edited by), La legge notarile, Milano, 2014, p. 823.

Protettì E., Di Zenzo C., La legge notarile, 5a ed., Milano, 2009, p. 474 ss.

Rampulla F.C., I principi generali della legge n. 241/1990 ed il responsabile del procedimento, in FA, 2008, 641.

Santarcangelo G., Il procedimento disciplinare a carico dei notai. Decreto legislativo 1 agosto 2006, Milano, 2007, passim.

Tenore V. e Celeste G., La responsabilità disciplinare del notaio ed il relativo procedimento, Milano, 2008, p. 10 ss. e p. 155 ss.

${ }^{47}$ F. Parente, La natura del procedimento dinanzi alla Co.Re.Di., cit., p. 826; M. Bove, Considerazioni in merito al procedimento disciplinare notarile, cit., p. 420. 


\section{Abstract}

Nel testo della legge 16 febbraio 1913, n. 89, il procedimento disciplinare notarile era modulato sulla diversificazione delle competenze secondo la gravità delle sanzioni.

Per le sanzioni disciplinari più gravi - l'ammenda, la sospensione e la destituzione - la competenza per i tre gradi di giudizio era affidata al giudice ordinario, ossia al Tribunale, alla Corte d'Appello e alla Corte di Cassazione.

Per le sanzioni meno gravi - l'avvertimento e la censura - la competenza, nel primo grado, era conferita al Consiglio notarile distrettuale, che decideva con provvedimento impugnabile dinanzi al giudice ordinario.

Il d.lgs. 1 agosto 2006, n. 249 ha modificato radicalmente il regime del procedimento disciplinare notarile e ha istituito la Commissione amministrativa regionale di disciplina (Co.Re.Di.), avente natura amministrativa, per l'applicazione delle sanzioni disciplinari e delle misure cautelari notarili e per la valutazione dei presupposti di cessazione temporanea o definitiva dalle funzioni notarili.

Nel quadro della riforma, invece, al Consiglio notarile distrettuale il legislatore ha assegnato il mero ruolo d'indagine e di controllo sul regolare svolgimento dell'attività notarile ed il potere di attivare il procedimento disciplinare tramite il diritto di azione accreditato al suo presidente

Parole chiave: notaio; procedimento disciplinare; natura giuridica.

\section{The notary disciplinary procedure and its historical evolution}

\section{Abstract}

In the law of 16 February 1913, no. 89, the notarial disciplinary procedure was modified introducing diversification of powers according to the severity of the penalties.

For the most serious disciplinary sanctions - fine, suspension and dismissal - the jurisdiction was assigned to the common courts, namely the Court of First Instance, the Court of Appeal and the Court of Cassation.

For the less serious penalties - warnings and censorships - the jurisdiction was conferred on the District Notary Council, which rulings could be challenged in front of common courts.

Dec. 1, 2006, no. 249 has radically altered the regime of the notarial disciplinary procedure and established the Regional Disciplinary Commission (Co.Re.Di.), which has an administrative nature, concerning the application of disciplinary sanctions and precautionary measures, as well as the assessment of the conditions of temporary or definitive termination of notarial functions.

In the framework of the reform, however, the District Notary Council was assigned the mere task of supervision and control over the regular conduct of the notary activity and the power to initiate disciplinary proceedings through the right of action was attributed to its chairman.

Keywords: notary; disciplinary proceedings; legal nature. 